\title{
Research on the Role of Th17Treg Cells and Their Factors in Graves' Disease with Different lodine Nutritional Status
}

\author{
Jing Feng ${ }^{1}$, Cuicui Wang ${ }^{2}$, Zhaoxin $\mathrm{Mu}^{2}$, Xinsheng $\mathrm{Li}^{1}$, Zhenjiang $\mathrm{Hou}^{2}$, * \\ ${ }^{1}$ Cangzhou Central Hospital, Cangzhou, China \\ ${ }^{2}$ Institute of Thyroid Diseases Affiliated to Cangzhou Medical College, Cangzhou Thyroid Disease Engineering Technology Research Center, \\ Cangzhou, China
}

Email address:

houzhenjiang@sina.com (Zhenjiang Hou)

${ }^{*}$ Corresponding author

\section{To cite this article:}

Jing Feng, Cuicui Wang, Zhaoxin Mu, Xinsheng Li, Zhenjiang Hou. Research on the Role of Th17Treg Cells and Their Factors in Graves' Disease with Different Iodine Nutritional Status. Science Journal of Public Health. Vol. 9, No. 1, 2021, pp. 1-11.

doi: $10.11648 /$ j.sjph.20210901.11

Received: December 18, 2020; Accepted: January 12, 2021; Published: January 18, 2021

\begin{abstract}
Introduction: At present, studies on the role of iodine nutrition in thyroid function stratification, antibody titer, Th17/Treg cells and related factors in the pathogenesis of GD have not been carried out. Objective: The acritical aims to investigate the correlation between thyroid function and autoantibody titers of Graves' disease (GD) patients with different iodine nutritional status with Th17/Treg cells, their cytokines and transcription factors, and the role of related factors in the pathogenesis of GD. Method: The levels of serum thyroid hormone, autoantibodies and urine iodine in 100 GD patients and 60 healthy subjects are detected by electrochemiluminescence instrument and iodine-catalyzed arsenic-cerium method, respectively. The ratio of Th17 cells to Treg cells and Th17/Treg ratio in peripheral blood mononuclear cells (PBMC) are detected by immunofluorescence-labeled monoclonal antibodies and flow cytometry. Real-time fluorescence quantitative PCR is used to detect the expression levels of retinoic acid-related orphan receptor (ROR- $\gamma \mathrm{t}$ ) and fork head/wing-shaped spiral transcription factor 3 (Foxp3) mRNA, and the serum IL-17 and TGF- $\beta$ levels are detected by ELISA. Result: As a result, the proportion of Th17 cells, serum IL-17 and ROR- $\gamma \mathrm{t}$ in PBMC of GD patients with different iodine nutritional status significantly increase, while the proportion of Treg cells, the expression of Foxp3mRNA and serum TGF- $\beta$ significantly decrease. The ratio of Th17/Treg cells in GD patients is significantly positively correlated with the titers of TPOAb and TgAb, and the titers of TPOAb and TgAb antibodies are significantly correlated with Th17/Treg, IL-17 and ROR- $\gamma$ t. Conclusion: In conclusion, thyroid hormones, autoantibodies, Th17, Treg cell ratios and dysfunctions as well as corresponding cytokines and transcription factors in GD patients with different iodine nutritional status participate in the development of GD.
\end{abstract}

Keywords: Iodine Nutritional Status, Graves' Disease, Th17 Cells and Treg cells, Cytokines and Transcription Factors

\section{Introduction}

Graves' Disease (GD), which is a common clinical endocrine disease and one of autoimmune thyroid disease (AITD), has the highest incidence among all types of hyperthyroidism. It accounts for about $85 \%$ of the causes of hyperthyroidism [1]. The prevalence of GD in Western countries is about $0.5 \%$ to $2 \%$, and the prevalence in our country is about $2 \%$ to $3 \%$ [2]. There are many factors in the pathogenesis of GD. Environmental, genetic, and autoimmune dysfunction can all lead to specific autoimmune reactions in the thyroid gland, producing thyroid peroxidase antibody (TPOAb) and thyroglobulin antibody (thyroglobulin antibody, $\mathrm{TgAb}$ ) autoantibodies, diffuse goiter, hyperthyroidism [3]. The clinical symptoms or signs of GD mainly include diffuse symmetrical goiter and hyperfunction, exophthalmos, and hypermetabolic state, etc., which can occur at any age, but it is more common in women. The adverse effects of this disease on multiple systems are very prominent, which can lead to multiple organ damage, poor treatment effects, and serious sequelae. In recent years, the incidence has been increasing, which seriously affects the quality of life of patients [4]. Research have found that there 
are many reasons leading to the increase in the incidence of GD, and iodine nutritional status may be one of the influencing factors. Excessive iodine intake increases the incidence of GD and the positive rate of thyroid autoantibodies, and as the disease worsens, the recurrence rate also increases [5].10-year changes before and after Universal Salt iodization (USI) indicate that the incidence of AITD increases with the increasing of iodine nutrition level. AITD is not only the main cause of abnormal thyroid function, it can also be combined with other AIDs to form multiple endocrine autoimmune syndromes, etc. It is also closely related to thyroid cancer and seriously endangers human health. A large number of epidemiological investigations have been carried out on the relationship between urinary iodine (UI), hyperthyroidism and GD, but the results of the research are not the same. Researches on AITD and Th1/Th2 subgroups have been extensively carried out in the past, but the traditional Th1/Th2 cell imbalance still cannot explain the pathogenesis of AITD well. Recent research has found that interleukin-17 (IL-17), transforming growth factor- $\beta$ (transforming growth factor, TGF- $\beta$ ) and retinoic acid-related orphan receptors (ROR- $\gamma \mathrm{t}$ ), Forkhead boxp3 (Foxp3) are the main cytokines and specific transcription factors secreted by Th17 and Treg cells, respectively. They play an important role in immunosuppression and have become the current occurrence of immune-related diseases. The research direction of development and outcome mechanism. In recent years, researches on Th17/Treg cells and ROR- $\gamma$ t and Foxp3 in RA and other AIDs have been widely carried out abroad [6], but there are not many domestic researches, and the proportion of Th17 and (or) Treg cells at home and abroad has a role in the pathogenesis of GD. The results of the research in GD are also inconsistent. The combined detection of Th17, Treg cells and ROR- $\gamma$ t, Foxp3, especially the relationship with GD, is rarely reported. Taking GD as the research object, there are few researches on the relationship between iodine nutrition level and thyroid function and autoantibody levels, especially research on the role of iodine nutrition status in the stratification of thyroid function and antibody titers and Th17/Treg cells and related factors in the pathogenesis of GD has not yet been carried out. The author carried out this research to explore the relationship between GD thyroid function stratification and antibody titers with different iodine nutritional status and Th17/Treg cells and related factors, which provides scientific basis for the development of precise treatment, prevention and pathogenesis of GD.

\section{Materials and Methods}

\subsection{Research Objects and Groups}

A total of 100 untreated inpatients with GD who are in the endocrinology department of Cangzhou Central Hospital and Cangzhou People's Hospital from March 2019 to August 2020 are selected as the experimental group, including 24 males and 76 females, with an average age (45.4 \pm 15.1$)$. A total of 60 healthy subjects with matching gender and age are selected as the control group (NC group), including 16 males and 44 females, with an average age of $(44.8 \pm 16.9)$ years. There are no statistically significant differences in gender and age in each group. All patients meet the diagnostic criteria of GD in the "Guidelines for the Diagnosis and Treatment of Thyroid Diseases in China" formulated by the Endocrinology Branch of the Chinese Medical Association in 2008 [7], and none of them have received Graves' disease related treatment. According to the results of thyroid hormone examination and whether there are corresponding clinical symptoms or signs, they are divided into GD-A group (clinical hyperthyroidism: with symptoms or signs of hyperthyroidism, while FT3 $>7.1 \mathrm{pmol} / \mathrm{L}, \quad$ FT4 $>22 \mathrm{pmol} / \mathrm{L}, \quad$ TSH $<0.27 \mathrm{mU} / \mathrm{L}$ ) and GD-B group (subclinical hyperthyroidism: no symptoms or signs of hyperthyroidism, FT3 and FT4 are normal, TSH $<0.27 \mathrm{mU} / \mathrm{L}$ ). Exclusion criteria: (1) Complicated with other autoimmune system diseases; (2) Acute and chronic infections, malignant tumors, pregnancy and allergic diseases; (3) Past history of diabetes, abnormal liver function and blood system diseases; (4) Those who have used glucocorticoid therapy in the past 30 days. The evaluation and analysis of iodine nutritional status adopt the standards issued by the World Health Organization (WHO), the United Nations Children's Fund (UNICEF) and the International Council for the Control of Iodine Deficiency Disorders (ICCIDD) in 2007. Median urinary iodine (MUI) $<100 \mu \mathrm{g} / \mathrm{L}$ is iodine deficiency, $100-199 \mu \mathrm{g} / \mathrm{L}$ is the right amount of iodine, $200-299 \mu \mathrm{g} / \mathrm{L}$ is iodine excess, $\geq 300 \mu \mathrm{g} / \mathrm{L}$ is iodine excess [8]. This research is approved by the ethics committee, and the research subjects sign an informed consent form.

\subsection{Research Methods and Projects}

\subsubsection{Specimen Collection and Processing}

(1) Specimen collection: All subjects are fasted for more than 12 hours, and collected $10-20 \mathrm{ml}$ of fasting mid-section urine in the morning, placed in a clean polyethylene plastic tube, stored in a refrigerator, and collected fasting venous blood at 8:00-10:00 5mL, after standing at room temperature for $1 \mathrm{~h}$, centrifuge at $3000 \mathrm{r} / \mathrm{min}$ for $10 \mathrm{~min}$, take serum, determine thyroid hormone content and autoantibody level. Avoid eating kelp, seaweed and other high-iodine foods and iodine-containing medicines, and avoid increasing drinking water for the first 1 day. (2) Peripheral blood mononuclear cell (PBMC) separation: Separate PBMC by Ficoll density gradient centrifugation, adjust the density of PBMC to $1 \times 10^{6} / \mathrm{L}$, and suspend it in the culture medium of RPMI640 fetal bovine serum. Place the PBMC suspension in a 24-well culture plate, add $25 \mu \mathrm{g} / \mathrm{L}$ of phorbol ester (Alexis, USA) and $1 \mathrm{mg} / \mathrm{L}$ of inomycin (Alexis, USA) to each well, incubate in a $37^{\circ} \mathrm{C}$ incubator for 4 hours, collect the cells stand-by.

\subsubsection{Project Testing}

(1) Use Cobas E601 immunochemiluminescence instru ment from Roche Co., Ltd. to determine serum thyroid stimulating hormone (TSH), total triiodothyronine (TT3), total thyroxine (TT4), free triiodothyronine (FT3), free $\mathrm{t}$ 
hyroxine (FT4), TPOAb, TGAb, the reagents are all orig inal Roche reagents. Due to regional differences and the influence of equipment, reagents and other factors, the reference value ranges of each hospital are different. Th e normal reference intervals for thyroid hormones and a utoantibodies in this hospital are as follows: TSH 0.27 $4.2 \mathrm{mIU} / \mathrm{L}$, FT3 $2.8 \sim 7.1 \mathrm{pmol} / \mathrm{L}$, FT4 12 22 $\mathrm{pmol} / \mathrm{L}, \mathrm{T} 3$ $1.3 \sim 3.10 \mathrm{nmol} / \mathrm{L}$, T4 66 181 nmol/L), TPOAb $0 \sim 34 \mathrm{IU} / \mathrm{m}$ 1, TgAb $0 \sim 115 \mathrm{IU} / \mathrm{ml}$. (2) Urine iodine determination ad opts the principle of iodine-catalyzed arsenic-cerium reac tion, and the urinary iodine content is detected by arseni c-cerium catalytic spectrophotometry (WS/T107-2006). Th e kit is produced by Wuhan Zhongsheng Biochemical Te chnology Co., Ltd., and the instrument is made by Hitac hi, Japan Model 7600-110 automatic biochemical analyze r. (3) Detection of the ratio of Th17 and Tregs cells: T h17 cells are labeled with a human Th17 cell detection kit (FITC/CD3, PE/CD4, Alexa Fluor647/IL-17, Biolegen $\mathrm{d}$, USA), and a human Treg cell detection kit (FOXP3 Alexa Fluor 488/CD4 PE-Cy5/CD25PE) to label Treg ce lls, use FACSCali-bur flow cytometer (BD company in $\mathrm{t}$ he United States), according to the instructions, add the corresponding fluorescent dye-labeled antibody into the fl ow tube containing $100 \mu \mathrm{L}$ of blood sample Incubate at $r$ oom temperature for 15 minutes in the dark, add red bl ood cell lysate, mix and incubate at $4^{\circ} \mathrm{C}$ for 10 minutes in the dark, add PBS solution and mix thoroughly and test on the machine. The test result is expressed as the percentage of positive cells and the fluorescence intensi ty of the positive cells, and the analysis data is obtaine $\mathrm{d}$ with CellQuest software.(4) Use real-time fluorescent quantitative PCR to detect the expression levels of ROR $-\gamma \mathrm{t}$ and Foxp3 mRNA: The total RNA of the sample is extracted by TRIzol method, and cDNA is synthesized a ccording to the reverse transcription procedure. Use Rev erse Transcriptase M-MLV for cDNA reverse transcriptio n, ROR- $\gamma \mathrm{t}$ upstream primer: 5'-AGTCCAGAACGAGGCA AAGC-3', ROR- $\gamma$ t downstream primer: 5'-CTTGG-CAAA CTCCACCACATA3'; Foxp3upstream primer 5'-ATTTTTA GGATTTGAGGTTTTAATA-3', Foxp3 downstream primer: 5'-CAAAAAACCCACTAAAAAACTATAC-3'. Real-time fluorescence quantitative PCR amplification is carried out using a fluorescence quantitative PCR instrument, accor ding to the following procedure: $95^{\circ} \mathrm{C}, 30$ seconds; 40 PCR cycles $\left(95^{\circ} \mathrm{C}, 5 \mathrm{~s}, 60^{\circ} \mathrm{C}, 40 \mathrm{~s}\right)$, and fluorescence is $\mathrm{c}$ ollected. After finishing the amplification reaction, press $95^{\circ} \mathrm{C}, 10 \mathrm{~s} ; 60^{\circ} \mathrm{C}, 60 \mathrm{~s} ; 95^{\circ} \mathrm{C}, 15 \mathrm{~s}$, the instrument will aut omatically proceed-Ramp Rate is $0.05^{\circ} \mathrm{C} / \mathrm{s}$, slowly heati ng from $60^{\circ} \mathrm{C}$ to $99^{\circ} \mathrm{C}$, strictly follow the product manu al for Real Time PCR samples are tested, each sample i $\mathrm{s}$ tested with 3 replicate wells, and the data is analyzed by the $2-\Delta \Delta C \mathrm{~T}$ method.(5) Detect serum IL-17 and TG F- $\beta$ levels by ELISA: The kit is provided by Biosource, and the operation is strictly in accordance with the kit instructions. Record the OD450 reading, using the OD v alue as the abscissa and the standard concentration as th e ordinate. Make a standard curve, and finally record th e levels of IL-17 and TGF- $\beta$.

\subsubsection{Statistical Methods}

Statistical methods Statistical analysis is carried out using SPSS 20.0 statistical software. Counting data is expressed as rate $(\%)$, and the comparison is made by $\chi^{2}$ test. Normal distribution measurement data is expressed as mean \pm standard deviation ( $\mathrm{x} \pm \mathrm{s}$ ), using the $\mathrm{t}$ test, with $\mathrm{P}<0.05$, which indicates that the difference is statistically significant. Non-normally distributed measurement data are described by median. Kruskal-Wallis $\mathrm{H}$ test is used for comparison among multiple groups, and Spearman is used for univariate correlation analysis. $\mathrm{P}<0.05$ is considered as statistically significant.

\section{Results}

\subsection{Comparison of Thyroid Hormone and Autoantibody Levels in GD Patients with Different Thyroid Functions}

100 cases of GD patients and GD-A, GD-B group thyroid hormone and autoantibody levels are compared. The results show that the serum TSH level of GD group, GD-A group, GD-B group is significantly lower than that of NC (all $\mathrm{P}<0.05)$. There is a statistically significant difference between GD-A and GD-B groups $(\mathrm{P}<0.05)$. With the hyperthyroidism of GD-B and GD-A, the TSH level gradually decreases $(\mathrm{P}<0.05)$. Serum FT3 and FT4 levels in GD, GD-A and GD-B groups are higher than $\mathrm{NC}$, but the difference between GD-B and NC groups is not statistically significant $(\mathrm{P}>0.05)$. There is no significant difference in serum T3 and T4 levels among the GD, GD-A and GD-B groups and the NC group $(\mathrm{P}>0.05)$. Serum $\mathrm{TPO}-\mathrm{Ab}$ and $\mathrm{TG}-\mathrm{Ab}$ levels in GD, GD-A and GD-B groups are significantly higher than $\mathrm{NC}(\mathrm{P}<0.01)$, and serum $\mathrm{TPO}-\mathrm{Ab}$ and TG-Ab levels in GD-A are also higher than those in GD-B group. See Table 1.

Table 1. Comparison of GD thyroid hormone and autoantibody levels with different thyroid functions.

\begin{tabular}{|c|c|c|c|c|c|c|c|c|}
\hline Groups & Num & TSH (mIU/L) & FT3 (pmol/L) & FT4 $(\mathrm{pmol} / \mathrm{L})$ & T3 (nmol/L) & T4 (nmol/L) & TPOAb IU/ml & TgAb IU/ml \\
\hline GD & 100 & $0.12 \pm 0.03 *$ & $34.52 \pm 5.81^{*}$ & $52.51 \pm 10.9^{*}$ & $1.81 \pm 0.76$ & $108.49 \pm 20.16$ & $386.5 \pm 184.9^{\mathbf{\Lambda}}$ & $247.13 \pm 70.2^{\mathbf{\Lambda}}$ \\
\hline GD-A & 81 & $0.09 \pm 0.06 *$ & $42.17 \pm 6.82 *$ & $64.13 \pm 12.4^{*}$ & $1.91 \pm 0.69$ & $110.32 \pm 18.75$ & $408.9 \pm 173.2^{\mathbf{\Lambda}}$ & $269.27 \pm 74.1^{\wedge}$ \\
\hline GD-B & 19 & $0.15 \pm 0.04 *$ & $5.35 \pm 0.62$ & $21.02 \pm 8.02$ & $1.79 \pm 0.82$ & $101.96 \pm 20.91$ & $359.2 \pm 179.6^{\mathbf{\Delta}}$ & $229.30 \pm 67.9^{\boldsymbol{\Lambda}}$ \\
\hline $\mathrm{NC}$ & 60 & $2.46 \pm 1.23$ & $4.91 \pm 0.58$ & $19.14 \pm 7.09$ & $1.82 \pm 0.42$ & $104.9 \pm 20.16$ & $9.16 \pm 1.02$ & $15.68 \pm 0.97$ \\
\hline
\end{tabular}

Note: Compared with the control group, * means $\mathrm{P}<0.05, \stackrel{\Delta}{ }$ means $\mathrm{P}<0.01$. 


\subsection{Comparison of Th17 and Treg Cells and Their Cytokines and Transcription Factors in GD Patients with Different Thyroid Functions}

Table 2. Comparison of Th17 and Treg cells and their cytokines and transcription factors in GD patients with different thyroid functions.

\begin{tabular}{|c|c|c|c|c|c|c|c|c|}
\hline Groups & Num & Th17cell\% & Tregcell\% & Th17/Treg & IL-17 (pg/ml) & TGF- $\beta(\mathrm{pg} / \mathrm{ml})$ & ROR- $\gamma t$ & Foxp3 \\
\hline G D & 100 & $3.54 \pm 0.61^{\boldsymbol{\Lambda}}$ & $3.19 \pm 1.14^{*}$ & $1.76 \pm 0.51^{\wedge}$ & $36.3 \pm 5.65^{*}$ & $14.09 \pm 4.71$ & $5.94 \pm 1.61^{*}$ & $1.76 \pm 0.24$ \\
\hline GD-A & 81 & $3.97 \pm 0.27^{\wedge}$ & $2.16 \pm 1.13 *$ & $1.84 \pm 0.02^{\Delta}$ & $37.3 \pm 4.94 *$ & $18.37 \pm 3.91$ & $7.37 \pm 0.41^{*}$ & $2.18 \pm 0.51 *$ \\
\hline GD-B & 19 & $3.16 \pm 0.31^{\boldsymbol{\Lambda}}$ & $3.09 \pm 1.04 *$ & $1.26 \pm 0.08^{\mathbf{\Delta}}$ & $28.9 \pm 3.12 *$ & $16.96 \pm 4.28$ & $3.87 \pm 0.32 *$ & $1.39 \pm 0.64^{*}$ \\
\hline $\mathrm{NC}$ & 60 & $0.91 \pm 0.28$ & $6.73 \pm 0.97$ & $0.12 \pm 0.04$ & $13.17 \pm 2.84$ & $15.97 \pm 3.68$ & $1.49 \pm 0.38$ & $1.89 \pm 0.63$ \\
\hline
\end{tabular}

Note: Compared with the control group, * is $\mathrm{P}<0.05$, and $\boldsymbol{\wedge}$ is $\mathrm{P}<0.01$.

The results of research find that the ratio of IL-17 cells, Th17/Treg ratio, IL-17 and ROR- $\gamma$ t in GD patients are higher than those in the $\mathrm{NC}$ group, which is gradually decreasing from HT-A to HT-B. The difference among the three groups is statistically significant $(\mathrm{P}<0.05)$. The proportion of Treg cells is significantly lower than that of the control group, which is gradually increasing from HT-A to HT-B. The levels of TGF- $\beta$ and Foxp3 are lower than those of the control group, which is gradually decreasing TGF- $\beta$ from HT-A to HT-B. The difference has not statistically significant, while Foxp3 gradually increases from HT-A to HT-B, and the difference has statistically significant $(\mathrm{P}<0.05)$.

\subsection{Comparison of Th17 and Treg Cells and Their Cytokines and Transcription Factors in GD Patients with Different Urine Iodine Levels}

With the different iodine nutritional status of GD patients who have iodine deficiency, iodine adequate, iodine excess, and iodine excess, the changes of Th17 and Tregs cells and their cytokines and transcription factors are basically consistent with the overall trend of GD patients. Among them, Th17 cells, the Th17/Treg ratio and ROR- $\gamma$ t increase significantly, but the differences among the groups are not statistically significant. See Table 3.

Table 3. Comparison of Th17 and Tregs cells and their cytokines and transcription factors in GD patients with different urine iodine levels.

\begin{tabular}{|c|c|c|c|c|c|c|c|c|}
\hline Group & Num & Th17Cell\% & TregCell\% & Th17/Treg & IL-17 (pg/ml) & TGF- $\beta$ & ROR- $\gamma \mathrm{t}$ & Foxp3 \\
\hline iodine deficiency & 11 & $3.46 \pm 0.47$ & $3.19 \pm 1.14$ & $1.14 \pm 0.39$ & $19.27 \pm 6.83$ & $12.9 \pm 4.71$ & $6.48 \pm 1.32$ & $1.70 \pm 0.28$ \\
\hline iodine adequate & 43 & $3.89 \pm 0.50$ & $2.74 \pm 0.61$ & $1.21 \pm 0.43$ & $22.92 \pm 7.06$ & $13.86 \pm 2.11$ & $6.91 \pm 1.09$ & $1.79 \pm 0.15$ \\
\hline iodine excess & 31 & $4.17 \pm 0.41$ & $2.68 \pm 0.34$ & $1.58 \pm 0.52$ & $24.08 \pm 7.15$ & $14.09 \pm 2.08$ & $7.32 \pm 1.15$ & $1.66 \pm 0.23$ \\
\hline iodine over-dose & 15 & $5.06 \pm 0.38$ & $2.89 \pm 0.42$ & $1.72 \pm 0.39$ & $23.17 \pm 6.36$ & $14.37 \pm 2.12$ & $7.86 \pm 0.97$ & $1.85 \pm 0.19$ \\
\hline
\end{tabular}

\subsection{Comparison of Th17 and Treg Cells and Their Cytokines and Transcription Factors in GD Patients with Different TPOAb and TgAb Levels}

The number of 100 GD patients with positive TPAAb and TGAb antibodies are 82 and 63, respectively, and the median antibody levels are $361.4 \mathrm{IU} / \mathrm{mL}$ and $238.6 \mathrm{IU} / \mathrm{mL}$, respectively. Using this as a critical value, GD is divided into high TPOAb group, low TPOAb group, high TGAb group, low TGAb group. Comparison of Th17 and Treg cells and their cytokines and transcription factors in GD patients with different TPOAb and TGAb levels showed that Th17 cells,
Th17/Treg, TGF- $\beta$ and ROR- $\gamma$ t in high-level TPOAb and $\mathrm{TgAb}$ groups are higher than low-level TPOAb. And the trend of $\mathrm{TgAb}$ group, among which Th17 cells and Th17/Treg are more obvious, but the difference is not statistically significant. In addition, the levels of Treg cells and IL-17 in the high-level TPOAb group are higher than those in the low-level TPOAb group, the levels of Treg cells and IL-17 in the high-level TgAb group are lower than those in the low-level TgAb group, and the Foxp3 level in the high-TgAb group is higher than that in the low-level TgAb group., But the differences are not statistically significant. See Table 4.

Table 4. Comparison of Th17 and Tregs cells and their cytokines and transcription factors in GD patients with different TPOAb and TGAb levels.

\begin{tabular}{|c|c|c|c|c|c|c|c|}
\hline Project & Th17Cell\% & TregCell\% & Th17/Treg & IL-17 (pg/ml) & TGF- $\beta$ & ROR- $\gamma \mathrm{t}$ & Foxp3 \\
\hline High TPOAb & $3.91 \pm 0.59$ & $2.98 \pm 1.02$ & $1.36 \pm 0.59$ & $23.1 \pm 6.14$ & $15.39 \pm 3.98$ & $6.53 \pm 1.49$ & $1.53 \pm 0.26$ \\
\hline Low TPOAb & $3.25 \pm 0.64$ & $2.53 \pm 1.26$ & $1.09 \pm 0.62$ & $22.9 \pm 5.96$ & $14.28 \pm 4.59$ & $5.46 \pm 1.82$ & $1.61 \pm 0.19$ \\
\hline High TGAb & $4.12 \pm 0.71$ & $2.87 \pm 1.32$ & $1.27 \pm 0.71$ & $21.3 \pm 6.08$ & $16.31 \pm 5.36$ & $6.37 \pm 1.57$ & $1.86 \pm 0.31$ \\
\hline Low TGAb & $3.84 \pm 0.49$ & $3.42 \pm 1.51$ & $0.98 \pm 0.58$ & $23.8 \pm 5.93$ & $14.62 \pm 5.07$ & $5.28 \pm 1.76$ & $1.69 \pm 0.24$ \\
\hline GD & $3.54 \pm 0.61$ & $3.19 \pm 1.14$ & $1.16 \pm 0.51$ & $21.3 \pm 5.65$ & $14.09 \pm 4.71$ & $5.94 \pm 1.61$ & $1.76 \pm 0.24$ \\
\hline
\end{tabular}

\section{Discussion}

Iodine is an essential element for the body to synthesize thyroid hormones. Its intake is an important factor affecting the changes in thyroid morphology and function. It has a "U" curve relationship with the occurrence of thyroid diseases.
That is, too much or too little iodine intake can lead to changes in the morphology and function of the thyroid gland, which have varying degrees of influence on the metabolism and function of almost all systems in the human body [9]. Iodine deficiency can increase the prevalence of nodular goiter, and excess iodine can increase the prevalence of hyperthyroidism, sporadic goiter and AITD [10]. At present, 
the incidence of various thyroid diseases is on the rise. The high incidence of thyroid diseases in the world is closely related to excess iodine nutrition. The relationship between high iodine and thyroid disease is the closest, and the high incidence of thyroid disease in my country is related to the excess iodine in the population or the increase in the number of individuals with excess iodine [11]. The MUI concentration of patients with thyroid disease is generally higher than that of the control. As the UI level increases, the incidence of thyroid disease increases, and the incidence is highest when the UI concentration reaches $300 \mu \mathrm{g} / \mathrm{L}$ and above [12]. The UI level of patients with thyroid disease $(306.9 \pm 116.2 \mu \mathrm{g} / \mathrm{L})$ is significantly higher than that of the control group $(195.7 \pm 101.1 \mu \mathrm{g} / \mathrm{L}, \mathrm{P}<0.01)$, which indicates that iodine excess is positively correlated with thyroid disease [13]. The mean values of MUI and UI from high to low are in the hyperthyroidism group (268.0 and $272.1 \pm 18.8 \mu \mathrm{g} / \mathrm{L})$, normal function group (199.7 and $203.7 \pm 16.2 \mu \mathrm{g} / \mathrm{L})$ and hypofunction group (177.6 and $185.6 \pm 19.3 \mu \mathrm{g} / \mathrm{L}$ ) [14]. The higher the UI level, the higher the thyroid function. The MUI of patients with thyroid disease $(324.56 \mu \mathrm{g} / \mathrm{L})$ is significantly higher than that of the control group $(167.98 \mu \mathrm{g} / \mathrm{L})$. Different types of thyroid diseases have the highest proportion of hyperthyroid UI overdose, while patients with iodine deficiency have the highest proportion of hyperthyroidism. The difference has statistically significant $(\mathrm{P}<0.05)$ [15]. Epidemiological survey data shows that after the implementation of universal salt iodization (USI), the incidence of hyperthyroidism has increased, and it mainly occurs in iodine-deficient areas [16]. Excessive iodine intake leads to excessive synthesis of thyroid hormones, which leads to the occurrence of hyperthyroidism [17]. Therefore, increased iodine intake is an independent risk factor for hyperthyroidism. GD has the highest incidence among all types of hyperthyroidism. Its clinical symptoms or signs mainly include diffuse symmetrical goiter, hyperfunction and hypermetabolic state, etc. It can occur at any age, but it is more common in women. In recent years, the incidence of GD has been on the rise, and there are many reasons for the increase in the incidence of GD, among which iodine nutrition status may be one of the influencing factors. In some countries with high iodine intake, such as Japan and the United States, the incidence of GD is significantly higher than that of iodine-adequate and iodine-deficient countries. A multi-center study in Austria finds that the incidence of GD and subclinical GD after the implementation of USI in iodine-deficient areas increases [18]. The prevalence of GD in the United States is $1 \%-2 \%$. The logistic regression analysis of the Graves' disease epidemiological survey of 100,000 people in Daqing area shows that the increase in iodine intake is closely related to the occurrence of GD and is an independent risk factor [19]. In 2010, a survey on the prevalence of thyroid disease among 15008 residents aged 15 and over in 10 cities in my country shows that the prevalence rates of hyperthyroidism, subclinical hyperthyroidism and Graves' disease are $0.89 \%, 0.72 \%$ and $0.61 \%$, respectively [20]. A survey of 56 members of
GD-prone families in 10 cities and counties in Liaoning Province find that the causes of thyroid dysfunction in first-degree relatives of GD-prone families are all AITD, and the autoantibody positive rate is $68.6 \%$. When $\mathrm{UI} \geq 500 \mu \mathrm{g} / \mathrm{L}$, the incidence of GD is significantly higher than that of groups with UI level $<500 \mu \mathrm{g} / \mathrm{L}$, and the differences are statistically significant $(\mathrm{P}<0.05)$, while, the difference between groups with UI below $500 \mu \mathrm{g} / \mathrm{L}$ is not statistically significant [21], which suggests that the increase in UI is a risk factor for the increased incidence of GD. The positive rates of TgAb, TPOAb and antibody titers in the high-iodine GD group are higher than those in the low-iodine GD group, and the difference is statistically significant $(\mathrm{P}<0.05)$ [5], which suggests that iodine excess significantly increase the autoantibody level of AITD patients. The prevalence rate of GD among residents in coastal areas of Shandong is $1.38 \%$, which is regionally distributed. The closer you are to the sea, the more iodine-containing foods you consume. The higher the UI level, the higher the prevalence rate of GD. The prevalence of GD with UI levels above $300 \mu \mathrm{g} / \mathrm{L}$ increases with the UI level [22]. The MUI $(302.95 \mu \mathrm{g} / \mathrm{L}, 354.4 \mu \mathrm{g} / \mathrm{L})$ of GD $(n=78)$ and HT $(n=31)$ patients is higher than that of the control group $(257 \mu \mathrm{g} / \mathrm{L})$, and the difference has statistically significant $(\mathrm{P}<0.0001)$ [23], and the UI level of HT patients is higher than that of GD patients, but the difference has not statistically significant $(\mathrm{P}=0.2078)$. It shows that the high iodine nutritional status in patients with AITD may be one of the reasons that lead to lipid peroxidation damage in the body and induce the development of AITD. The above research results indicate that the increasing in iodine intake is closely related to the occurrence of GD and the production of thyroid autoantibodies, and is a risk factor for the increase in the incidence of GD.

The results of research on urine iodine, thyroid hormone and autoantibody levels of GD patients are also different. The MUI of GD patients in the coastal area of western Guangdong is above $300 \mu \mathrm{g} / \mathrm{L}$. The median of the two autoantibodies are higher than the other groups, and the difference among the four groups of different UI levels has statistically significant $(\mathrm{P}<0.05)$, while there has no statistically significant difference among FT3, FT4, and TSH groups [24]. A research on the clinical characteristics of 210 GD patients find that the higher the TPOAb titer, the more obvious the degree of goiter [25]. Goiter of GD patients is related to age and high titers of TPOAb and TRAb. FT3, FT4, $\mathrm{TPOAb}$ and TRAb in the goiter group are higher than those in the no goiter group $(\mathrm{P}<0.05)$. Thyroid eye signs are related to high titers of TgAb and TRAb [26]. The untreated group is the highest, followed by the uncontrolled group, and the difference has statistically significant. After 131 I treatment for 3 months, the level of IL-17 is the lowest, which is slightly higher than the control group, but the difference has not statistically significant. The level of serum IL-17 in GD patients changes positively with the concentration of FT3, FT4, and TRAb, while it changes negatively with TSH [27]. The Th17 cell ratio and serum IL-17 and TGF- $\beta 1$ levels of patients in GD and remission groups are significantly higher 
than those in the control group, and are positively correlated with FT3, FT4, and TRAb, but negatively correlated with TSH [28]. GD patients ( $n=20)$ FT3, FT4 are significantly higher than the NC group, while TSH is significantly lower than the NC group $(\mathrm{P}<0.05)$, and the GD eye disease $(\mathrm{GO})$ group FT3, FT4 and TPOAb, TgAbare significantly higher than the GD group $(\mathrm{P}<0.05)$. The TPOAb and TgAb in the GD group are also significantly higher than those in the NC group, and the difference between the three groups has statistically significant $(\mathrm{P}<0.05)$ [29]. GD patients $(\mathrm{n}=65)$ FT3, FT4, TPOAb, and TgAb all increase significantly, while TSH decrease [30]. The titers of TgAb and TPOAb in HT patients $(n=36)$ are significantly higher than those in GD patients $(n=48)$, and GD patients are higher than those in the control group, and the difference has statistically significant $(\mathrm{P}<0.01)$. The TPOAb titer of GD patients is significantly lower than that of HT patients, but the difference in $\mathrm{TgAb}$ titer between the two groups has not statistically significant. The FT3 and FT4 of GD patients are higher than the control group, and TSH is lower than the control group. TSH in HT patients is significantly higher than that in the control group, while FT3 and FT4 are not statistically different from the control group [30]. The titers of TgAb and TPOAb in GD, HT, and hypothyroidism groups are significantly higher than those in the control group, and the GD group is higher than the HT group, while there has no statistically significant difference between TSH in HT patients and the control group [31]. Serum TgAb and TPOAb titers of HT and GD patients are significantly higher than those of the control group, and the HT group is higher than the GD group, the difference has statistically significant [32]. There are also reports that the titers of TPOAb and TgAb in HT and GD patients are higher than those in the control group, but there is no statistically significant difference between the two groups [33]. Spearman correlation analysis shows that MUI in patients with hyperthyroidism is positively correlated with TSH and negatively correlated with FT3. MUI in patients with subthyroidismis negatively correlated with FT4, and MUI in patients with hypothyroidism is positively correlated with TPOAb titers, which suggests that there are differences in UI levels in patients with different types of thyroid diseases. UI is related to thyroid hormone levels and related antibodies [7] The above research results confirm that the titers of $\mathrm{TgAb}$ and TPOAb in HT and GD patients are significantly higher than those in the control group, but there are differences in antibodies between the two groups and the results of FT3, FT4 and TSH are not the same, which may be related to the composition ratio and severity of the disease, Staging and gender differences.

The results of this research are basically consistent with most of the above-mentioned studies. As the level of UI increases, TSH, TPOAb, and TgAb in GD patients increase, while FT3, FT4, TT3, and TT4 decrease. The effect of high iodine intake on TSH of GD patients is significantly better than that of FT3 and FT4, but has no effect on T3 and T4. Therefore, thyroid function indicators (FT3, FT4, TSH), especially TSH changes, combined with GD patients' TgAb titers are significantly higher than HT patients, and HT patients TPOAb titers are significantly higher than GD patients. And the higher the UI level, the higher the autoantibody titer. Combined detection is helpful for the diagnosis and differential diagnosis of GD and HT, and has certain value for the efficacy and prognosis judgment. The results of this research also show that the composition ratio of GD patients with different UI levels is from high to low, in order of iodine adequate, iodine excess, iodine beyond, and iodine deficiency. The sum of iodine excess and iodine beyond is consistent with the proportion of the iodine adequate group, which is significantly higher than that of the iodine deficiency group, and the MUI of the GD-A group is significantly higher than that of the GD-B group, which indicates that high iodine can easily induce the occurrence of GD. The results of most studies are basically the same.

Th17/Treg cells are new types of immune cells discovered in recent years. They have independent differentiation and regulation mechanisms and inhibit each other. Both of them and their cytokines play an important role in the pathogenesis of AITD [34]. Most research believe that the pathogenesis of AITD is the result of the combined effects of environment, genetics, and immune imbalance, among which immune factors are recognized as the main cause of disease. Th17/Treg is a pair of Th cell subsets with pro-inflammatory and anti-inflammatory effects. The former mediates inflammatory response by secreting Th17 cytokines, while the latter exerts immunosuppressive effects through direct contact and secretion of cytokines. Foxp3 regulates the activation process of effector $\mathrm{T}$ cells at the transcriptional level. Previous research has found that there is a certain correlation between AITD and Th17/Treg cell imbalance, cytokines and thyroid autoantibodies, but the results of the studies are not the same. The proportion of Treg cells in the thyroid tissue and PBMC of AITD patients is significantly higher than that in the control group, and there are functional defects. Their immunosuppressive ability significantly reduces, and the body's immune response cannot be effectively down-regulated to prevent the occurrence of AITD [35]. The thyroid tissue of GD patients and the control group almost does not express IL-17, and the expression of IL-17mRNA in PBMC is low, while IL-17 is strongly positive in HT patients, and the level of IL-17mRNA is elevated [36]. Serum IL-17 levels in GD and HT patients are significantly higher than those in the control group, with HT being more pronounced, which indicates that Th17 cells in GD patients have a lower ability to produce IL-17 factor than HT. AITD patients $(n=49)$ Th17 cells and IL-17, IL-6, TGF- $\beta$ are higher than the control group, especially HT patients Th17 cells and IL-17 increase more significantly. The proportion of Th17 cells is positively correlated with IL-17 levels, but has no correlation with IL-6 [37]. Zhang et al. reports that the proportion of Th17 cells in GD patients significantly increases, while Treg cells significantly reduce, which indicates that Th17 and Treg cells play an important role in the pathogenesis of GD [38]. Li et al. find that Th17 cells in PBMC of GD patients and serum IL-17 levels of 
refractory GD patients significantly increase, while $\mathrm{CD}^{+}{ }^{+} \mathrm{FoxP}^{+} \mathrm{T}$ (Treg) and its transcription factor FoxP3 mRNA content significantly decrease, and IL-17 levels in remission patients have no significant changes comparing with the control group. The proportion of Treg cells in GD patients is lower than that in the control group, and Foxp3 expresses at a low level on the surface of Treg cells [39]. The proportion of Th17 cells in GD patients $(n=50)$ is higher than that in the control group, while $\mathrm{CD} 4{ }^{+} \mathrm{CD} 25^{+} \mathrm{FoxP} 3^{+} \mathrm{T}$ cells and Treg cells are significantly lower than that in the control group [40]. The proportion of Th17 cells and serum IL-17 levels in GD patients $(n=40)$ PBMC are higher than those in the control group, while the proportion of $\mathrm{CD} 4{ }^{+} \mathrm{CD} 25^{+}$Tregs cells and serum TGF- $\beta$ levels are lower than those in the control group $(\mathrm{P}<0.05)$ [41]. The $\mathrm{CD} 4^{+} \mathrm{CD} 25^{+}$Treg in the PBMC of GO patients is significantly lower than that of the GD group, and the GD group is significantly lower than that of the NC group. The difference among the three groups has statistically significant $(\mathrm{P}<0.05)$ [42]. There is no significant correlation between $\mathrm{Th} 17 / \mathrm{CD}^{+} \mathrm{T}$ cells in PBMC of GD patients and thyroid autoantibodies. The levels of Th17 cells and IL-17 in PBMC of HT and GD patients and TGF- $\beta 1$ levels in HT patients are significantly higher than those in the control group $(\mathrm{P}<0.05)$. TGF- $\beta 1$ levels in HT patients are significantly higher than those in the control and GD groups, while the level of TGF- $\beta 1$ in GD patients is not significantly different from that in the control group [43], which suggests that Th17 cells and their cytokines IL-17 and TGF- $\beta 1$ may be involved in the pathogenesis of AITD, and TGF- $\beta 1$ may play a greater role in the pathogenesis of HT, but has a smaller role in the pathogenesis of GD. The number of $\mathrm{CD}^{+} \mathrm{T}$ cells and the proportion of $\mathrm{CD} 4^{+} \mathrm{CD} 25^{+}$Tregs in $\mathrm{CD} 4^{+} \mathrm{T}$ cells in the PBMC of GD $(n=40)$ and HT patients are not significantly different from those of the control group $(\mathrm{P}<0.05)$, while the expression level of TGF- $\beta 1$ and Foxp3mRNA significantly decrease [44]. The ratio of $\mathrm{CD} 4{ }^{+} \mathrm{CD} 25^{+}$Foxp $3^{+}$Treg cells/CD4 ${ }^{+} \mathrm{T}$ cells in PBMC of AITD patients is significantly lower than that of GD and HT patients with normal thyroid function. The number of $\mathrm{CD} 4{ }^{+} \mathrm{CD} 25^{+}$Treg cells in AITD patients decrease, especially Foxp $3^{+} \mathrm{CD} 4^{+} \mathrm{CD} 25^{+}$Treg cells are more obviously, and the activated $\mathrm{CD}^{+}{ }^{+} \mathrm{T}$ cells increase [45]. The proportion of $\mathrm{CD} 4^{+} \mathrm{CD} 25^{+}$Treg in the PBMC of 50 $\mathrm{HT}$ and GD patients in $\mathrm{CD} 4^{+} \mathrm{T}$ cells and the expression level of Foxp3 mRNA are significantly lower than those of the control group (all $\mathrm{P}<0.05$ ), while the serum TGF $\beta 1$ level is significantly higher than that of the control group (all $\mathrm{P}<0.05$ ) [46]. $\mathrm{CD}^{+} \mathrm{IL} 17^{+} / \mathrm{CD}^{+} \mathrm{CD} 25^{+} \mathrm{CD} 127^{-}$and $\mathrm{CD} 4^{+} \mathrm{IL} 17^{+}$in PBMC of GD patients/CD $4^{+} \mathrm{CD} 25^{+} \mathrm{CD} 127^{-}$.

FoxP $3^{+} \mathrm{T}$ cells are significantly lower than the control group, and show a positive change with the change of TRAb [26]. The expression levels of FoxP3, IL-10 mRNA and their protein in GD patients significantly increase. After IL-21 stimulation, the above indicators significantly reduce, and there is no significant difference compared with the control group after treatment recovery [47]. Th17 cell ratio, Th17/Treg ratio, and ROR- $\gamma \mathrm{t}$ and IL-17 gene expression levels significantly increase in GD patients, while Treg and
Breg ratios and Foxp3 gene expression levels significantly decrease, but IL-10 gene expression levels do not change significantly [48]. CD $4^{+}$Foxp $3^{+}$cells, $\mathrm{CD} 4^{+} \mathrm{CD} 25^{+} \mathrm{CD} 127$ (low) Foxp $3^{+} \mathrm{T}$ cell frequency and $\mathrm{CD} 4^{+} \mathrm{CD} 25^{+} \mathrm{CD} 127$ (low) Foxp $3^{+} / \mathrm{CD}^{+} \mathrm{CD} 25$ (high) in GD patients. The proportion of CD127 (low) $\mathrm{T}$ cells is significantly lower than that of patients with nodular goiter [49]. The expression levels of FoxP3 and IL-10 mRNA and IL-10 protein in the PBMC of GD patients are significantly higher than those of the remission group and the control group, while the expression levels of IL-17, ROR- $\gamma$ t, IL-22 mRNA and IL-22 protein between the GD remission group and the control group are not statistically different [50]. Most of the above research results show that the proportion of Treg cells and TGF- $\beta$ levels in GD patients reduce, but there are also reports that the proportion of Treg cells is not statistically different from the control group [51] and higher than the control group [34]. The results of this research show that the proportion of IL-17 cells, Th17/Treg ratio, IL-17 and ROR- $\gamma$ t in GD patients are all higher than those in the NC group, and gradually decrease from HT-A to HT-B. The differences among the three groups have statistically significant $(\mathrm{P}<0.05)$. The ratio of Th17 cells and Th17/Treg increase most significantly. The ratio of Treg cells is significantly lower than that of the control group, and gradually increased from HT-A to HT-B. The levels of TGF- $\beta$ and Foxp3 are lower than those of the control group. $-\mathrm{A}$ to HT-B gradually decrease, and the difference has not statistically significant, while Foxp3 is gradually increased from HT-A to HT-B, and the difference has statistically significant $(\mathrm{P}<0.05)$.

GD is one of the most common organ-specific AITDs and the most common clinical type of hyperthyroidism. Its etiology involves many factors such as environment and genetics. Its pathogenesis has not yet been fully elucidated [52] and has always been a focus of research in this field. Research have found that the onset of GD is related to urine iodine, thyroid function and autoantibody titers. The results of this research show that TPOAb and TgAb titers in GD patients increase with the progression of the disease and the increase in UI levels, and the urinary iodine level is positively correlated with TPOAb and TgAb titers, which is consistent with the view that thyroid autoantibodies can aggravate immune damage [53], which indicates that high iodine may be the main environmental factor that induces the occurrence and development of GD. Excessive iodine intake can not only promote the occurrence of thyroid autoimmune reactions, but also further accelerate the occurrence of abnormal thyroid function in patients with abnormal thyroid autoimmunity. It may be related to the fact that high iodine can aggravate iodine's organic barriers, reduce thyroid hormone synthesis, and promote AIT in people with autoimmune background; Excessive iodine leads to thyroid tissue damage, which can accelerate the exposure of thyroid antigens, activate autoimmune responses, and induce the occurrence and development of GD [54]. The results of this research also show that the ratio of Th17/Treg cells in GD patients is significantly positively correlated with the titers of 
TPOAb and TgAb, and the antibody titers of TPOAb and TgAbare significantly correlated with Th17/Treg, IL-17 and ROR- $\gamma$ t. Th17/Treg cell imbalance may play a role in the production of thyroid autoantibodies and the abnormal $\mathrm{T}$ cell immune response mediated by them, thereby participating in the immune damage of thyroid tissue itself. However, the interaction and mechanism of different iodine nutritional status, thyroid function, autoantibodies and Th17/Treg cells and their factors in the pathogenesis of GD remain to be studied in depth.

Previous research has found that organ-specific AID is mainly related to Th1 cells. With the deepening of research on $\mathrm{T}$ cell subgroups, the understanding of autoimmunity has changed significantly [55]. Recent research has found that Th17 and Treg cells play an important role in the pathogenesis of AITD [56]. Th17 and Treg cells are CD4 ${ }^{+} \mathrm{T}$ cell subgroups with opposite effects. The former mainly mediates inflammation through secretion of high levels of IL-17 and other cytokines, and the latter mainly through direct cell contact and secretion of inhibitory cytokines (TGF). $-\beta$, IL-10, etc.) play an immunosuppressive function, and both and their cytokines play an important role in the pathogenesis of AID [57]. Researches in recent years have confirmed that Th17, Treg cells and their related factors are related to the occurrence of multiple AIDs. Foxp3 can be specifically expressed in $\mathrm{CD} 4^{+} \mathrm{CD} 25^{+} \mathrm{Foxp}^{+}$Treg. Foxp $3^{+}$ plays a prominent role in $\mathrm{CD} 4^{+} \mathrm{CD} 25^{+}$in regulating the development and functional expression of $\mathrm{T}$ cells. $\mathrm{CD} 4^{+} \mathrm{CD} 25^{+} \mathrm{Foxp}^{+}$Treg cells can also be used in a variety of ways. Immune suppression is an important mechanism for the stability of the immune system itself [58]. Foxp $3^{+}$Treg of $\mathrm{CD} 4^{+} \mathrm{CD} 25^{+}$in GD patients significantly decreased, while the proportion of $\mathrm{CD} 4^{+} \mathrm{CD} 25^{+} \mathrm{T}$ cells does not change significantly. It is suggested that the lack of immunosuppressive Foxp $3^{+}$Treg be closely related to the pathogenesis of GD. The detection of Foxp3 expression level may be more meaningful than the change in the proportion of $\mathrm{CD} 4{ }^{+} \mathrm{CD} 25^{+}$cells. There is no significant change in the number of Tregs in GD patients, but the expression of Foxp3 decreases. Therefore, it is believed that the onset of GD is mainly caused by Treg functional defects [59]. Regardless of whether the proportion of Treg cells in GD patients is normal or not, their immunosuppressive ability significantly decreases, indicating that abnormal Treg cell function is closely related to the pathogenesis of GD. The proportion of $\mathrm{CD} 4^{+} \mathrm{CD} 25^{+} \mathrm{Foxp}^{+}$Treg in $\mathrm{CD} 4^{+} \mathrm{CD} 25^{+} \mathrm{T}$ cells and $\mathrm{CD} 4^{+} \mathrm{T}$ cells in GD patients is lower than that in the control group, which indicates that the reduction of $\mathrm{CD} 4^{+} \mathrm{CD} 25^{+}$Foxp $3^{+}$Treg cells may play an important role in the occurrence of GD. In vitro experiments show that $\mathrm{CD} 4{ }^{+} \mathrm{CD} 25^{+} \mathrm{Foxp} 3^{+}$Treg cells can significantly inhibit the proliferation of effector $\mathrm{T}$ cells, and affect the autoimmune system in many ways, and have an impact on the process of GD multiple autoimmune pathology. The results of this research show that the Th17 cell ratio, Th17/Treg ratio, serum IL-17 and ROR- $\gamma \mathrm{t}$ in PBMC of GD patients significantly increase, while the Treg cell ratio, Th17/Treg ratio and Foxp3 mRNA expression level significantly decrease. The increase in Th17 cell ratio and the decrease in Treg cell ratio in the PBMC of patients with hyperfunction are more obvious than those in patients with normal thyroid function and subclinical hyperthyroidism, which manifests as progressive changes in different stages of the disease, which indicates that abnormal thyroid function may affect the function of Th17/Treg cells, Th17/Treg cell imbalance runs through different stages of GD, consistent with the results of Xue Haibo and Pyzik [60, 61] in HT changes, but the degree of change is not as obvious as HT. It is confirmed that different thyroid function itself may have a certain impact on Th17 and Treg cells in PBMC, and the imbalance between the two may play an important role in the pathogenesis of GD [62]. It is speculated that the changes in the proportion of Th17 and Treg cells in GD patients at different stages may be caused by changes in thyroid function. Whether it is directly related to the immune response needs further investigation.

Th17/Treg cells participate in the autoimmune damage of thyroid tissue through the abnormal immune response of $\mathrm{T}$ cells mediated by TGF- $\beta$ and other regulatory factors [63]. Treg cells mainly exert their biological effects by secreting TGF- $\beta$ and IL-10 [64]. TGF- $\beta$, as the main cytokine secreted by Treg cells, has different effects on the development of human Th17 and Treg cells. The development of Treg cells and the expression of the key transcription factor Foxp3 depend on the existence of TGF- $\beta$, while the development of Th17 cells does not depend on TGF- $\beta$ [65]. Researches in TGF- $\beta 1$ deficient mouse models have confirmed that TGF- $\beta 1$ plays an important role in maintaining peripheral $\mathrm{CD} 4^{+} \mathrm{CD} 25^{+}$Treg expression Foxp3 and exerting immunosuppressive effects. High concentrations of TGF- $\beta 1$ can up-regulate the expression of Foxp3, induce the conversion of human $\mathrm{CD} 4^{+} \mathrm{CD} 25^{-}$Treg into $\mathrm{CD} 4^{+} \mathrm{CD} 25^{+}$Treg, and express Foxp3, which exerts a function similar to the naturally developed Treg cells in the thymus. The results of this research show that the proportion of Th17 cells, serum IL-17 and ROR- $\gamma$ t in PBMC of GD patients significantly increase, while the proportion of Treg cells and Foxp3 mRNA expression levels significantly decrease, and serum TGF- $\beta$ decreased. It shows that the reduction of Treg cells reduces the immunosuppressive ability of effector $\mathrm{T}$ cells, causing the body to appear immune disorders, leading to the disappearance of immune tolerance and the destruction of immune homeostasis, the activation of immune function, the activation of autoantibodies, and the induction of AID. Foxp3 can inhibit the transcription of IL-17A mRNA by directly binding to ROR- $\gamma \mathrm{t}$, thereby affecting the function of Th17 cells. At the same time, the low level of Foxp3 attenuates the inhibition of ROR- $\gamma \mathrm{t}$ expression, cannot effectively promote the development of Treg cells, does not respond to TGF- $\beta$, prompts Th17 cells to escape the immunosuppressive effect of Treg cells, and many other mechanisms jointly participate in Th17/Treg of GD patients The imbalance of cell subpopulations may play an important role in the pathogenesis of GD.

The above research results show that the thyroid function, 
autoantibody titers, Th17/Tregs cells and their cytokines IL-17 and TGF- $\beta$, transcription factors ROR- $\gamma \mathrm{t}$ and Foxp3 mRNA under different iodine nutrition conditions have corresponding changes in GD. The balance of Th17/Treg cells can maintain the body in a steady state of immune tolerance. In the case of inflammation or autoimmune diseases, Th17 cell differentiation is enhanced and Treg cell differentiation is inhibited, which destroys the Th17/Treg cell balance or leads to the body's Damage [65]. Th17 cell ratio, IL-17 level increase and Treg cell ratio decrease, especially $\mathrm{CD} 4{ }^{+} \mathrm{CD} 25^{+} \mathrm{FoxP}^{+}$Treg cell decrease or Treg cell dysfunction, may play a synergistic role in the pathogenesis of GD, and jointly participate in the pathogenesis of GD, but the change is not as obvious as in HT. It shows that the ratio of Th17/Treg cells and its factors cooperate in the immune response of the body, leading to changes in thyroid function and the occurrence of GD, but its mechanism of action is still unclear.

\section{Conclusion}

The results of this research show that the titers of TPOAb and $\mathrm{TgAb}$ in GD patients are significantly higher than those in the control group, which increase with the progression of the disease and the increase in UI levels. The effect of high-level antibodies on thyroid hormones is significantly better than that of low-level antibodies, and the level of urine iodineis positively correlated with TPOAb and TgAb titers, that is, the higher the urine iodine level, the more obvious the changes in thyroid hormones, and the higher the level of autoantibodies. Serum TSH levels in the high TPOAb group and high TgAb group are lower than those in the low TPOAb group, low $\mathrm{TgAb}$ group, and control group, respectively. The levels of FT3 and FT4 are higher than those in the low TPOAb group and low TgAb group, but the low TgAb FT3 is compared with the control group and the high TPOAb. There is no statistically significant difference in T3 and T4 levels between the high TgAb group, the low TPOAb group and the low TgAb group. This is consistent with the view that thyroid autoantibodies can aggravate immune damage, which indicates that high iodine may be the main environmental factor that induces the development of GD. The proportion of Th17 cells, serum IL-17 and ROR- $\gamma$ t in GD patients' PBMC significantly increase, while the proportion of Treg cells and Foxp3 mRNA expression significantly decrease, and serum TGF- $\beta$ decrease. The ratio of Th17/Treg cells in GD patients is significantly positively correlated with the titers of TPOAb and $\mathrm{TgAb}$, and the titers of TPOAb and TgAb antibodies have a greater correlation with Th17/Treg, IL-17 and ROR- $\gamma$ t It shows that Th17/Treg cell immune imbalance and the changes of its factors may play a role in the production of thyroid autoantibodies and the abnormal $\mathrm{T}$ cell immune response mediated by them, thereby participating in the immune damage of thyroid tissue itself. It is suggested that abnormalities of urine iodine level, thyroid function, TPOAb, $\mathrm{TgAb}$ and Th17/Treg cells and related factors are involved in the development of GD. Carrying out multi-center and large-sample prospective researches to explore the interaction and mechanism of different iodine nutritional status, thyroid function stratification, autoantibodies and Th17/Treg cells and their factors in the pathogenesis of GD, which will open up new ways for precise prevention and treatment of GD.

\section{Acknowledgements}

Cangzhou Key R \& D Plan and Guidance Project (Project No: 1833020011).

Natural Science Foundation of Cangzhou Medical College (No. 18Z015).

\section{References}

[1] Chen X, Mei Y, He B, et al. General and specific genetic polymorphism of cytokines-related gene in AITD [J]. Mediators Inflamm, 2017, 2017 (1): 3916395.

[2] XueL, PanC, GuZ, et al. Genetic heterogeneity of susceptibility gene in different ethnic populations: refining association study of PTPN22 for Graves' disease in a Chinese Han population [J]. PLoS One. 2013, 8 (12): e84514.

[3] Li HX, Xiang N, Hu WK, et al. Relation between therapy options for Graves' disease and the course of Graves'ophthalmopathy: a systematic review and meta-analysis [J]. J EndocrinolInvest, 2016, 39 (11): 1225-33.

[4] Burch HB, Cooper DS. Management of Graves' disease: A Review [J]. JAMA, 2015, 314 (23): 2544-2554.

[5] Su JP, Su SO, Zhang B, et al. The effects of different amounts of iodine in take on the immunestatus of patients with Graves' disease [J]. Clin Med China, 2012, 28 (1): 44-46.

[6] Hou ZJ, Mu ZX, Wang CC. Research Progress of Th17/Treg Cells and Their Transcription Factors in Autoimmune Diseases [J]. American Journal of Clinical and Experimental Medicine 2019, 7 (4): 83-92.

[7] Chinese Medical Association Endocrine Credits "China thyroid disease treatment guidelines" Writing Group. Chinese Guidelines for the Diagnosis and Treatment of thyroid diseases-Hyperthyroidism [J]. ChinJ Intern Med, 2007, 46 (10): 876-882.

[8] Chinese Medical Association Endocrine Credits "China thyroid disease treatment guidelines"Writing Group. China thyroid disease treatment guidelines-iodine deficiency disorders [J]. ChinJ Intern Med, 2008, 47 (8): 689-690.

[9] Tao LY, Li X, Huang J, et al. Correlative studies of iodine on thyroid nodular diseases [J]. 2013, 23 (27): 82-85.

[10] Jiang HX, Li YB. Progress of the influence of iodine on thyroid disease and its mechanism [J]. J Chin Pract Diagn Ther, 2016, 30 (7): 639-641.

[11] Chen ZX, Xu WM, Huang YM, etal. Associations of noniodized salt and thyroid nodule among the Chinese population: a large cross-sectional study [J]. Am J Clin Nutr. 2013, 98 (3): 684-692.

[12] Fei L, Zhang YL, Wang PY, et al. Analysis of urinary iodinelevels in patients with thyroiddiseases in Yuhuan county [J]. Chin JEpidem, 2010, 31 (2): 239-240. 
[13] Wu ZF, Liang Y, Chen XQ. Investigation of urinary iodine in patients with thyroid disease [J]. Youjiang-medi, 2010, 38 (2): 188-189.

[14] Huang SY, Yan YM, Wu M, et al. Analysis of Urinary Iodine Content of Newly Diagnosed Thyroid Disease Patients in Xiaogan [J]. Guangzhou Microelement Science 2015, 22 (1): 24-27.

[15] Xu L, Chen WH. Analysis of the difference of urinary iodine levels among patients with different types of thyroid diseases [J] J Anhui Med, 2020, 14 (7): 785-787.

[16] Fan X, Chen SK. Relationship of Iodine Excessiveness and Thyroid Function [J]. Med Reca, 2011, 17 (14): 2165-2167.

[17] Tan X, Cao XX, He L. Effect of iodine excess on thyroid function and its pathogenesis of diseases [J]. Chin J Ctrl Endem Dis, 2019, 34 (1): 35-37.

[18] Yang F, Teng WP, Shan ZY, et al. A comparative epidemiologic survey of hyperthyroidism in areas with different iodine intake [J]. Chin J Endocrinol Metab, 2001, 17 (4): 197-201.

[19] Li CQ, Yin HQ, Zhang CK, et al. Epidemiological investigation of Graves's disease in one hundred thousand peoplein Daqing area $[\mathrm{J}]$. National Medical Journal of China, 1996, 76 (6): 443-446.

[20] Shan Z, Chen L, Lian X, et al. Iodine status and prevalence of thyroid disorders after introduction of mandatory universal saltiodization for 16 years in China: a crosssectional study in 10cities [J]. Thyroid 2016, 26 (8): 1125-1130.

[21] Jin Y, Teng WP, Yuan B, et al. Thyroid autoimmunity in members from multiplex families with Graves' disease and effect of iodine intake on its incidence [J] Chin J Endocrinol Metab, 2001, 17 (2): 79-82.

[22] Yan SL, Wang YG, Wang F, et al. Relationship between iodine in urine and Graves's disease along coastal district in shading [J]. Chin J Endem, 2004, 23 (3): 245-247.

[23] Cong JN, Zhao YJ, Li G, et al. The changes of SOD and MDA in serum of patients with autoimmune thyroid disease and the relationship between SOD and urine iodine Guangzhou Microelement Science, 2011, 18 (8): 12-15.

[24] Chen XM, Guo ZQ, Zhou F, et al. Correlation of concentrations of iodine in urine with Graves' disease along coastal area of western Guangdong [J]. China Tropi Med, 2010, 10 (8): 922-923.

[25] Chen XY, Zhang ZT, Deng SY, et al. Clinical manifestation of Graves' disease patients with different levels of thyroid related autoantibodies [J]. New Med, 2011, 42 (12): 789-792.

[26] Huang YZ, Tang BY, Chang L, et al. Influencing factors on goiter and thyroid eye in patients with Graves' disease [J] J Anhui Med, 2015, 36 (5): 580-582.

[27] Li L, Liu YM, Chen TS, et al. Expression of serum IL-17 in Graves' disease patients [J]. J Guangdong Pharm Univ, 2014, 30 (3): 363-365.

[28] Li HL, Deng ZK, Zheng YH, et al. Expression of Th17 cells and related cytokines in peripheral blood of patients with Graves' disease [J]. J Immun Chin 2016, 32 (4): 563-566.

[29] Ding JY, Qin LD, Xu Y, et al. Expression and Significance of
PD-1/PD-L1 on $\mathrm{CD}^{+} \mathrm{CD} 25^{+}$Treg Cells in Peripheral Blood of Patients with Graves' Disease [J]. Prog Mode Biom, 2019, 19 (4): 718-723.

[30] Ma, JG, Wei HL, Shang H. Correlational between peripheral blood $\mathrm{T}$ cell subsets and thyroid function and thyroid autoantibodies in patients with Graves' disease [J]. Int J Endocrinol Metab, 2020, 40 (1): 21-26.

[31] Huang J, Yu PF, Yang ZP. Clinical application of thyroid-stimulating hormone and thyroid autoantibodies in the diagnosis of thyroid disease [J]. Chin J Ctrl Endem Dis, 2015, 30 (1): 65-66.

[32] Dong X. Diagnostic value of serum thyroid autoantibodies in patients with autoimmune thyroid disease [J] Clin Med, 2018, 38 (5): 40-41.

[33] Yang J, Li Q. Clinical application of thyroid stimulating hormone and thyroid autoantibodies in the diagnosis of thyroid diseases [J]. Proc Clin Med, 2018, 27 (6): 424-426.

[34] Bossowski A, Moniuszko M, Idkowska E, et al. Decreased proportions of $\mathrm{CD} 4{ }^{+} \mathrm{IL} 17^{+} / \mathrm{CD} 4^{+} \mathrm{CD} 25^{+} \mathrm{CD} 127^{-}$and $\mathrm{CD} 4^{+} \mathrm{IL} 17^{+} / \mathrm{CD}^{+} \mathrm{CD} 25^{+} \mathrm{CD} 127^{-} \mathrm{FoxP} 3^{+} \mathrm{T}$ cells in children with autoimmune thyroid diseases [J]. Auto-immunity, 2016, 49 (5): 320-328.

[35] Marazuela M, García-López MA, Figueroavega N, et al. Regulatory T Cells in Human Autoimmune Thyroid Disease [J]. J Clin Endocrinol Metab, 2006, 91 (9): 3639-3646.

[36] Figueroa-Vega N, Alfonso-Pérez M, Benedicto I, et al. Increased circulating pro-inflammatory cytokines and Th17 lymphocytes in Hashimoto's thyroiditis [J]. J Clin Endocrinol Metab, 2010, 95 (2): 953-962.

[37] Chen ZJ, Liu C, Li Q, et al. The change of Th17 lymphocytes and cytokines in autoimmune thyroid diseases [J]. Immu J, 2011, 27 (9): 785-788.

[38] Zhang DH, Qiu XG, Li JH, et al. MiR-23a-3p-regulated abnormal acetylation of FoxP3 induces regulatory $\mathrm{T}$ cell function defect in Graves' disease [J]. Biol Chem. 2019, 24; 400 (5): 639-650.

[39] Li JR, Hong FY, Zeng JY, et al. Functional interleukin-17 receptor A are present in the thyroid gland in intractable Graves' disease [J]. Cell Immunol. 2013, 281 (1): 85-90.

[40] Zhou XJ, Li WP. Relationship between $\mathrm{CD}^{+} \mathrm{CD} 25^{+} \mathrm{FoxP}^{+} \mathrm{T}$ cell regulatory T cells and Th17 cells and Graves' disease [J]. J Taishan Medical College, 2019, 40 (12): 907-908.

[41] Li CH, Chen ZL, Sun QK, et al. The mechanistic study of $\mathrm{CD} 4^{+} \mathrm{CD} 25^{+}$regulatory $\mathrm{T}$ and $\mathrm{Th} 17$ cells on Graves disease $[\mathrm{J}]$. Northern Pharm, 2015, 12 (5): 123-124.

[42] Zheng L, Chen ZJ, Liu C. Changes of Th3 and Th17 lymphocytes and related cytokines in patients with autoimmune thyroid disease [J]. China J Immu, 2013, 29 (1): 43-47.

[43] Gao ST, Zhu TN, Zhang YN, et al. The frequency and function of $\mathrm{CD}^{+} \mathrm{CD} 25^{+}$regulatory $\mathrm{T}$ cells in autoimmune thyroid diseases [J]. China J Immu, 2011, 27 (3): 269-273.

[44] Mao C, Wang S, Xiao Y, et al. Impairment of regulatory capacity of $\mathrm{CD} 4{ }^{+} \mathrm{CD} 25^{+}$regulatory $\mathrm{T}$ cells mediated by dendritic cell polarization and hyperthyroidism in Graves' disease [J]. JImmunol. 2011, 186 (8): 4734-4743. 
[45] Zheng LT, Yu SP, Wang N, et al. Regulatory $\mathrm{T}$ cells and TGF- $\beta 1$ in autoimmunetherole of thyroid disease [J]. Shandong Med 2012, 52 (1): 81-82.

[46] Huang XQ, Liu C. Effect of IL-21 on Treg cells in peripheral blood mononuclear cells in pathogenesis of Graves' disease [J]. Chin J Immu, 2016, 32 (6): 853-857, 862.

[47] Qin J, Zhou J, Fan CL, et al. Increased Circulating Th17 but Decreased CD $4{ }^{+}$Foxp $3{ }^{+}$Treg and $\mathrm{CD} 19^{+} \mathrm{CD} 1 \mathrm{~d}$ hi $\mathrm{CD} 5{ }^{+} \mathrm{Breg}$ Subsets in New-Onset Graves' Disease [J]. Biomed Res Int. 2017, 2017: 8431838.

[48] Pawlowski P, Grubczak K, Kostecki J, et al. Decreased Frequencies of Peripheral Blood $\mathrm{CD} 4^{+} \mathrm{CD} 25^{+} \mathrm{CD}$. 127 ${ }^{-} \mathrm{Foxp}^{+}$.

in Patients with Graves' Disease and Graves' Orbitopathy: Enhancing effect of Insulin Growth Factor-1 on Treg Cells [J]. HormMetab Res, 2017, 49 (3): 185-191.

[49] Tan Y, Chen W, Liu C, et al. Effect of IL-21 on the Balance of Th17 Cells/Treg Cells in the Pathogenesis of Graves' Disease [J]. Endocr Res. 2019, 44 (4): 138-147.

[50] Glick AB, Wodzinski A, Fu P, et al. Impairment of regulatory T-cell function in autoimmune thyroid disease [J]. Thyroid, 2013, 23 (7): 871-878.

[51] Du J, Wang X, Tan GQ, et al. Research progress on the Graves' disease susceptibility genes [J]. Chin J Comp Med, 2019, 29 (4): 126-132.

[52] Caturegli P, De Remigis A, Chuang K, et al. Hashimoto's thyroiditis: celebrating the centennial through the lens of the Johns Hopkins hospital surgical pathology records [J]. Thyroid. 2013, 23 (2): 142-150.

[53] Latrofa F, Fiore E, Rago T, et al. Iodine contributes to thyroid autoimmunity in humans by unmasking a cryptic epitope on thyroglobulin [J]. J Clin Endocrinol Metab, 2013, 98 (11): E1768-1774.

[54] Zhu JF, Yamane H, Paul WE. Differentiation of effector CD4 T cell populations [J]. Annu Rev Immunol. 2010, 28: 445-489.
[55] Gonza'lez-Amaro R, Marazuela M. T regulatory (Treg) and T helper 17 (Th17) lymphocytes in thyroid autoimmunity [J]. Endocrine, 2015, 52 (1): 1-9.

[56] Kleinewietfeld M, Hafler DA. The plasticity of human Treg and Th17 cells and its role in autoimmunity [J]. Semin Immunol. 2013, 25 (4): 305-312.

[57] Huang F, Bi JH, Hao LX, et al. Detection and clinical significance of peripheral blood $\mathrm{CD} 4{ }^{+} \mathrm{CD} 25^{+}$Foxp $3^{+}$regulatory $\mathrm{t}$ cells and urinary iodine in patients with Graves's disease [J]. HeBei Med, 2017, 23 (1): 147-149.

[58] Wang H, Zhao S, Tang X, et al. Changes of regulatory T cells in Graves' disease [J]. Huazhong Univ Sci Technolog Med Sci, 2006, 26 (5): 545-547.

[59] Xue HB, Ma L, Li YB, et al. Correlation between Treg/Th17 cells imbalance andautoimmunity in Hashi-moto's thyroiditis [J]. Chin JMode Med, 2012, 22 (23): 67-71.

[60] PyzikA, GrywalskaE, Matyjaszek-MatuszekB, et al. Immune disorders in Hashimoto's thyroidditis: what do we know so far? [J]. J Immunol Res. 2015, 2015: 979167.

[61] González-AmaroR, Marazuela M. T regulatory (Treg) and T helper17 (Th17) lymphocytes in thyroid autoimmunity [J]. Endocrine. 2016, 52 (1): 30-38.

[62] Li C, Ebert PJ, Li QJ. T cell receptor (TCR) and transforming growth factor $\beta$ (TGF- $\beta$ ) signaling converge on DNA (cytosine-5)-methyltransferase to control forkhead box protein 3 (foxp3) locus methyl-and inducible regulatory $\mathrm{T}$ cell differentiation [J]. J Biol Chem. 2013, 288 (26): 19127-19139.

[63] Cai H, Kong W, Zhou T, et al. Radiofrequency ablation versus reresection in treating recurrent hepatocellular carcinoma: a meta-analysis [J]. Medicine, 2014, 93 (22): e122

[64] Hirota K, Martin B, Veldhoen M. Development, regulation and functional capacities of Th17 cells [J]. Sem in Immunopathol, 2010, 32 (1): 3-16.

[65] Khan U, Ghazanfar H. T Lymphocytes and Autoimmunity [J]. Int Rev Cell Mol Biol. 2018, 341: 125-168. 